\title{
Performance Evaluation of Microservices Architectures using Containers
}

\author{
Marcelo Amaral, Jordà Polo, David Carrera \\ Technical University of Catalonia (UPC) \\ Barcelona Supercomputing Center (BSC)
}

Email: marcelo.amaral, jorda.polo, david.carrera@bsc.es

\author{
Iqbal Mohomed, Merve Unuvar, \\ Malgorzata Steinder \\ IBM T.J. Watson Research Center, Yorktown Heights, NY
}

Email: iqbal, munuvar, steinder@us.ibm.com

\begin{abstract}
Microservices architecture has started a new trend for application development for a number of reasons: (1) to reduce complexity by using tiny services; (2) to scale, remove and deploy parts of the system easily; (3) to improve flexibility to use different frameworks and tools; (4) to increase the overall scalability; and (5) to improve the resilience of the system. Containers have empowered the usage of microservices architectures by being lightweight, providing fast start-up times, and having a low overhead. Containers can be used to develop applications based on monolithic architectures where the whole system runs inside a single container or inside a microservices architecture where one or few processes run inside the containers. Two models can be used to implement a microservices architecture using containers: master-slave, or nested-container. The goal of this work is to compare the performance of CPU and network running benchmarks in the two aforementioned models of microservices architecture hence provide a benchmark analysis guidance for system designers.
\end{abstract}

Keywords-Containers, Networking, Performance Evaluation, Microservices.

\section{INTRODUCTION}

Virtual Machines are a widely used building block of workload management and deployment. They are heavily used in both traditional data center environments and clouds (private, public and hybrid clouds). The commonly used term Virtual Machine (VM) refers to server virtualization, which can be accomplished via full virtualization or paravirtualization. In recent months, there has been a resurgence of interest in container technology, which provides a more lightweight mechanism - operating system level virtualization. Containers are lightweight and fast - a single x86 server can reasonably have 100s of containers running (memory usually ends up being the scarce resource); moreover, containers start up very quickly - under 1 to 2 seconds in most cases. There are many reasons for this resurgence but from a technical perspective, two of the biggest reasons are (i) the improvements in namespace support in the Linux kernel are available in popular distributions, and (ii) a specific implementation of containers - Docker - has successfully created an attractive packaging format, useful tools and diverse ecosystem.

Containers are operating-system-level virtualization under kernel Linux that can isolate and control resources for a set of processes. Because of container does not emulate a full virtualization of the physical hardware as VM does, it is lightweight with less overhead. The core of containers rely on Linux namespace [1] and cGroups [2]. The former is an abstraction that wraps a set of processes appearing that they are isolated instance. Linux namespace isolates the set of filesystem mount points seen by the group of processes. cGroups organize the processes in a hierarchy tree; they also limit, police and account the resource usage of process group. One can run a single application within a container whose namespaces are isolated from other processes on the system. Notwithstanding, the main capability of container is to allow to run a complete copy of the Linux OS within it without the overhead of running hypervisor. Although the kernel is shared, they have limited access to the modules and drivers to the ones that it has leaded. Despite a container has limited access, it can have full access to the host when created as "privileged". Such a privileged container might run also another daemon inside to create other containers as a nested-container approach; but can emerge security problems when sharing the infrastructure with other users. Currently, there exist many container distributions such as OpenVZ [3], and Docker [4].

At a high-level, current Docker usage can be categorized into two classes: (i) Docker container as a lightweight server, and (ii) one process (or few related processes) per Docker container. There have been extensive studies on (i), such as [5]; in this paper, we concern ourselves with (ii). To our knowledge, this approach (related processes per container) does not have a widely used name even though the technique itself is common. For purposes of this paper, we will call it Related Processes Per Container or RPPC. RPPC is sensible from the perspective of deployment, and is also a useful building block in microservices architectures. We explain both in turn.

Consider a traditional application server that implements some business logic and talks to a remote database server. Along with the core application server, one would install auxiliary software or sidecars that provide facilities such as logging, performance monitoring, configuration management, proxying, and so on. Rather than packaging all of this software into a single unit, one can group related processes in containers and then deploy the ensemble. When some functionality needs to be updated, one need only deploy a subset of the original containers. Thus, the RPPC approach typically speeds up deployment, reduces disruption and generally empowers the devops team. It should be noted that this concept is not new to even containers - configuration management tools such as Puppet and Chef have provided this capability for years - only 
update what needs to be changed. What's different is that individual containers are the unit of deployment.

The RPPC concept is also useful as a building block for microservices. Microservices is a new trend in architecting large software systems wherein a system is designed as a set (dozens or even hundreds) of microservices. Microservices can be developed, managed and scaled independently. There is typically some kind of routing fabric that gets requests to a specific instance of a microservices; this routing fabric often provides load-balancing and can isolate microservices that are in a failed state. One system that provides these capabilities in a cloud or clustered environment is Google's Kubernetes. In Kubernetes, a pod is a group of containers that is a deployable unit - moreover, all containers of a pod share the same fate. While this doesn't always make sense (e.g. in the application server example above), it does make a lot of sense in microservices and especially with containers (destroying a faulty pod does no harm since the routing fabric will route to healthy one; also, since starting up containers is a lot faster than booting Virtual Machines, pod startup can be very fast).

We investigated two distinct ways to implement the RPPC concept. In the first approach, all child containers are peers of each other and a parent container (which serves to manage them). We call this implementation as master-slave throughout the paper. The second approach, where we refer as nestedcontainer, involves the parent container being a privileged container and the child containers being inside its namespace. Our purpose is to understand the performance differences of the two approaches hence system designers can benefit from our analysis in the future.

\section{MICROSERVICES ARCHITECTURE USING CONTAINERS}

\section{A. Containers}

Containers are a mechanism that provide operating system level virtualization, in that they can isolate and control resources for a set of processes. Because a container does not emulate the physical hardware as a virtual machine does, it is lightweight with less overhead. While the concept of operating system level virtualization is not new (e.g. chroot/jails in BSD), there has been a great deal of industry interest in Linux containers and Docker Inc's implementation in particular. The core of containers rely on Linux namespaces [1] and cGroups [2]. Linux namespaces isolate the set of filesystem mount points seen by a group of processes. cGroups organize the processes in a hierarchy tree; it also limits, polices and accounts for the resource usage of process groups. One can run a single application within a container whose namespaces are isolated from other processes on the system. On the other hand, one can use a container as a lightweight Linux server, by setting up facilities such as a process control system (e.g. supervisord) and and ssh server. Currently, there exist many container implementations such as OpenVZ [3], Rocket [6] and Docker [4]. In this paper, we primarily focus on Docker containers.

Normally, a Docker container has limited access to resource on the host, but full access can be provided by creating it as a "privileged" container. Such a privileged container can access host devices and also run another Docker daemon inside itself to create child containers (we call this the nested-container approach). One must be careful with this approach as security challenges may emerge when sharing system resources with other users.

\section{B. Key virtual networking differences}

In recent years, there have been significant advances in virtual networking features in Linux. Some notable mechanisms include network namespaces, veth pairs, tap devices as well as virtual switches such as OpenvSwitch and Linux Bridges. The mechanisms can be used to provide a high degree of flexibility and control of networking, and form the basis of SDN (software defined networking) technologies such as Openstack Neutron. For instance, a physical host might have just one physical network interface, while a number of guest containers can run in isolation by having their own network namespaces, with tap devices wired into an OpenvSwitch. Moreover, one could setup tunnels between the OpenvSwitch instances on different machines, and can enable communication between instances. In practice, there are a great many ways of setting up virtual networking, with varying effects on throughput, latency and CPU utilization.

In this paper, we mainly focus our attention to communication that takes place on a single host. Even in this restricted case, there are many options to consider. Figure 2 shows 4 different communication setups that we explore later in the paper, along with the various layers that must be traversed. Of course, best performance is achieved when a process runs on bare-metal and can utilize the native networking stack in the OS. The default setup with Docker is that containers are wired into a virtual switch such as a Linux Bridge or OpenvSwitch (which is wired into the physical NIC/OS networking stack). In the nested-container scenario, there is a second level of virtual switching that takes place inside the container. In the virtual machine case, there is typically a virtual switch as well as virtualized network drivers.

\section{Microservices}

Microservices have gained much popularity in industry in the last few years. This architecture can be considered a refinement and simplification of Service-oriented Architecture (SOA) [7]. The key idea is that rather than architecting monolithic applications, one can achieve a plethora of benefits by creating many independent services that work together in concert. The benefits accrued include simpler codebases for individual services, ability to update and scale the services in isolation, enabling services to be written in different languages if desired and utilize varying middleware stacks and even data tiers for different services [8]. There are costs to this approach as well [9] such as the computational overhead of running an application in different processes and having to pay network communication costs rather than simply making function calls within a process. The proliferation of service processes almost requires automated mechanisms for deployment (sometimes called continuous delivery or continuous integration). Other challenges include deciding what the boundaries of different services are and determining when a service is too big.

\section{Achieving Microservices with Containers}

As previously stated, Docker containers are an excellent match for building microservices. They are lightweight, start 
very fast, and can wrap dependencies and vagaries of implementation inside themselves. For instance, a developer can start dozens of containers on a modest laptop of the day. They can go to a source such as DockerHub to download images of containers with pre-configured middleware, databases and applications. Such images can be extended with additional customization of the developer's choosing.

When one considers how services developed as ensembles of containers can be deployed, two different approaches emerge: master-slave or nested-containers as illustrated in Figure 1.

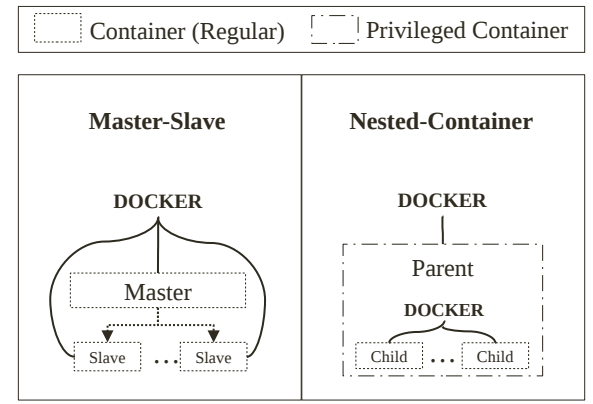

Fig. 1. Overview of master-slave and nested-container models.

The master-slave is composed by one container as the master coordinating other containers called slaves, in which the application process will be running. In this approach the master needs to track the subordinates' containers, help their communication and guarantee that the slaves do not interact with other containers from a different master (in this paper, in order to simplify, we refer to the master-slave approach as regular-container). On the other hand, in the nested-container approach, the subordinates' containers (the children) are hierarchically created into the main container (parent). The parent might be completely agnostic and just exist. The children run the application process and they are limited by the parent's boundaries. The nested-containers approach might be easier to manage since all other containers are inside only one container. This approach also might benefit from easily performing IPC, guaranteeing fate sharing, and sharing the same memory, disk and network. But, nested-containers approach might include more overhead than the master-slave approach, since it has two layers of Docker daemon as illustrated in Figure 2.

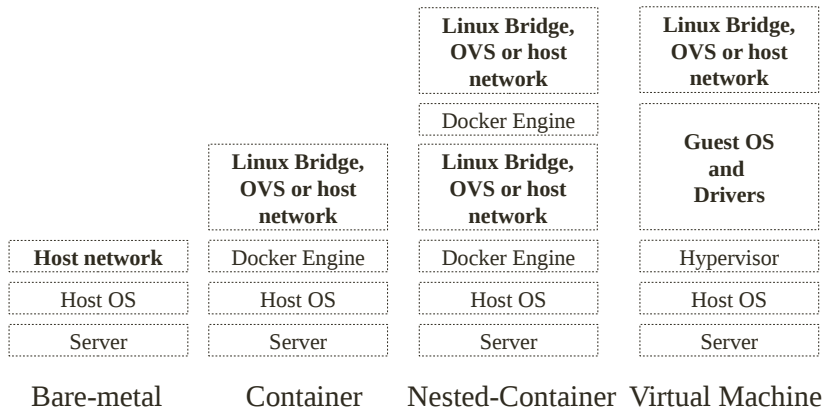

Fig. 2. The network stack for bare-metal, container, nested-container and virtual-machine.

\section{RELATED WORK}

To the best of our knowledge, this is the first work to analyze performances of microservices architecture using containers as nested and master-slave implementations. However, there is an extensive work that has been done in the area of performance comparison between virtual machines and Linux containers [5]. Most of the work in the literature is comparing hypervisors to the other hypervisors or to non-virtualized environments [10], [11].

Virtual machines were first introduced by IBM on mainframes in 1970s [12] and since then reinvented by many companies such as VMware, Xen and KVM. Linux containers have a long history as well. Linux-V Server project [13] was the first attempt by Linux to implement virtual private servers. In 2007, Linux introduced native containers which brought us today's containerization technology.

The most popular container implementation, as of writing this paper, is Docker [4]. It is an open platform that has been used for the last couple of years for developers and system administrators to build, ship and run the applications without the VM overhead [4]. Docker containers have been widely accepted and used by the open source cloud community and the enterprises. Recently, IBM started an engagement with the Docker community [14] and built their Beta version of container cloud [15]. Companies like VMware, Warden Container, Imctfy are also focusing on building container clouds as the containerization technology rapidly gains popularity.

The significance of comparing virtualization and containerization is twofold. One hand, virtualization helps isolation with the cost of latency and overhead. And, on the other hand containerization brings less overhead along with a similar but less isolation than virtual machines. In terms of isolation capabilities of containers, [16] studied performance of different container implementations such as Linux, Docker, Warden Container, Imctfy and OpenVZ along with virtual machines. As for the trade-off between performance and isolation between containers and virtual machines, [17] examined number of experiments for High Performance Computing environments. Performance of network virtualization for containers is also well studied in the Linux literature [18] however not in the nested, master-slave container, virtual machines or baremetal level.

Nested-containers are inspired from the "pod" concept that is implemented by Google for better managing Docker containers [19]. Pods are the smallest deployable group of containers that are placed on the same host. However, the performance of pods over virtual machines or bare-metal instances has not been studied. The contribution of our paper is to provide benchmark analysis for container virtualization via implementing nested and master-slave containers hence comparing the performance against virtual machines and baremetal.

Microservices management gained popularity over the last years. Google Cloud Platform implemented and open sourced a pre-production Beta cluster management project -Kubernetesthat can be used for better management of large scale microservices [20]. Kubernetes provides lightweight, simple and self-healing services management. With its high scalability and simplicity properties, Kubernetes promotes container technology via microservices architecture. Mesos [21] is another open source project that is intended to be an operating system for a datacenter. Mesos is built similar to the Linux kernel with a different level of abstraction. Mesos is composed of master(s), slave(s) and framework(s). Frameworks are designed 
to manage the different type of workloads hence Mesos provide a hierarchical resource management solution that increases the scalability and reduces the latency due to resource scheduling. Mesos is highly scalable $(10,000$ s of nodes). This makes Mesos a strong open source resource management tool for microservices management. [22] studied the performance of Mesos on multiple types of cluster computing such as Hadoop and MPI however did not consider the container technology.

\section{EVALUATION}

In order to explore the performance of the two containerbased environments discussed in Section II, we execute five different kinds of experiments mostly focused on CPU and network performance. In Experiment 1 (Section IV-B), we consider the execution of a CPU-intensive benchmark in order to verify whether there is any performance difference between the studied approaches. In Experiment 2 (Section IV-C), we evaluate the overhead of virtual container creation that might be used for management decisions. In Experiment 3 (Section IV-D), we evaluate the overhead of creating nestedcontainers. In Experiment 4 (Section IV-E), we compare the proposed approaches, but with a focus on network performance of local traffic on one host. Finally, in Experiment 5 (Section IV-F), we also evaluate the network, but with a focus on remote traffic across two hosts.

The main goal of these experiments is to study the performance and overhead of nested-containers, which might play a key role in the implementation of microservices architectures. In all experiments we compare the performance of the following environments: i) bare-metal ii) regular containers (representing the master-slave approach) iii) nested-containers iv) virtual machines.

\section{A. Evaluation infrastructure}

The machines that are used to run the experiments are several 2-way Intel Xeon E5-2630L, each one composed by 2 sockets, 6 cores per socket, 2 hyper-threads per core at $2 \mathrm{GHz}, 64 \mathrm{~GB}$ of RAM and 1 Gbps Network Interface Card (NIC). They are Linux box running Ubuntu 14.04 (Trusty Tahr) with Linux Kernel 3.13. Those machines are in the same rack and are connected with 2 stacked Gigabit Cisco Switch model 3750X with 48port each, connected through StackWise+ connector. For containers we used Docker 1.0.1 build 990021a, while virtualization was provided by KVM 2.0.0 configured with Intel Virtualization Technology (VT$\mathrm{x}$ ) and network Gigabit mode (virtio). Experiments focused on CPU (IV-C and IV-D) are based on the Sysbench benchmark [23] version 0.4.12. Experiments focused on network (IV-E and IV-F) use Netperf version 2.6.0, and the machines are configured with Open vSwitch version 2.0.2, and the Linux Bridge version natively available in Ubuntu 14.04. All the programs were compiled using gec version 4.8.2 and Python 2.7.6.

\section{B. Experiment 1: CPU Performance Evaluation}

The goal of this first experiment is to compare the computing performance of different kinds of environments: bare-metal, regular containers, nested-containers, and virtual machines. In order to evaluate the performance, we selected the Sysbench benchmark [23] running in CPU mode, where each request calculates prime numbers up to a certain value specified by the cpu-max-primes option, in this experiment set to 40,000; all calculations are performed using 64-bit integers.

This experiment measures the mean execution time of running Sysbench, increasing the number of concurrent Sysbench instances, from 1 up to 64. For bare-metal, we simply run multiple Sysbench instances on the host machine. For containers, we execute multiple containers, each one running a single Sysbench instance. And for virtual machines, we also run multiple virtual machines executing a single Sysbench instance. No resource constraints are set of containers or virtual machines, so the scalability is expected to grow linearly with the number of available CPU cores.

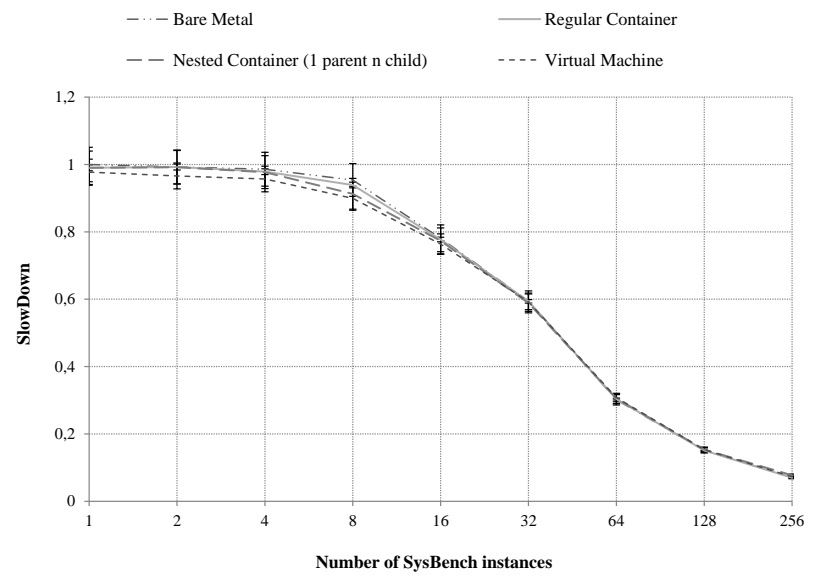

Fig. 3. Observed slowdown of Sysbench with increasing number of instances relative to running a single Sysbench instance in bare-metal

The results of this experiment are illustrated in Figure 3, which shows the observed slowdown of running Sysbench when increasing the number of instances. In particular, the Figure shows the average of 10 executions for each one of the tested environments: bare-metal, regular containers, nestedcontainers, and virtual machines. The baseline to measure the slowdown is the execution time of one single Sysbench instance running on bare-metal.

As it can be observed in Figure 3, all environments display a similar behavior, confirming there is no significant performance impact for CPU-intensive executions when running on containers or virtual machines compared to bare-metal. Containers run natively in the operating system, and they are only isolated by a lightweight layer (cgroups and namespaces), so as expected they basically perform as well as bare-metal. But even virtual machines perform as much thanks to improved virtualization support in modern processors. It should also be noted that slowdown degrades slowly up to 8-16 concurrent instances, and significantly faster after that. This is basically related to the characteristics of the experimental machine, which has 12 cores. When more than 12 instances are running, they have to share the same cores, leading to increased context switching.

\section{Experiment 2: Comparing Overhead of Virtual Container Creation}

While there is no significant performance impact for CPU bound applications under different environments, there may 
be a higher variation when considering the management of the proposed approaches.

This experiment evaluates the performance impact of creating the hosting entities for different virtualization technologies: containers, nested-containers (Microservices), and virtual machines in the context of server virtualization. The goal of this experiment is to evaluate the scalability of managing different kinds of virtual containers.

We measure the time to create an increasing number concurrent hosting entities (containers or virtual machines) for each one of the proposed approaches, from 1 up to 64 . Each hosting entity simply launches a dummy application that takes a negligible amount of time (in particular, we used Sysbench as in Experiment 1, this time configured with cpu-max-primes set to 1), effectively allowing us to compare creation times under different environments. For regular containers, we measure elapsed time between container startup and exit. For nested-containers, we measure the elapsed time between starting-up and exiting the parent container, which also includes loading a locally-stored child image as well as starting-up and exiting a single child container. Finally, for a virtual machine, we measure the time to create a virtual machine domain, start the domain and delete the domain.

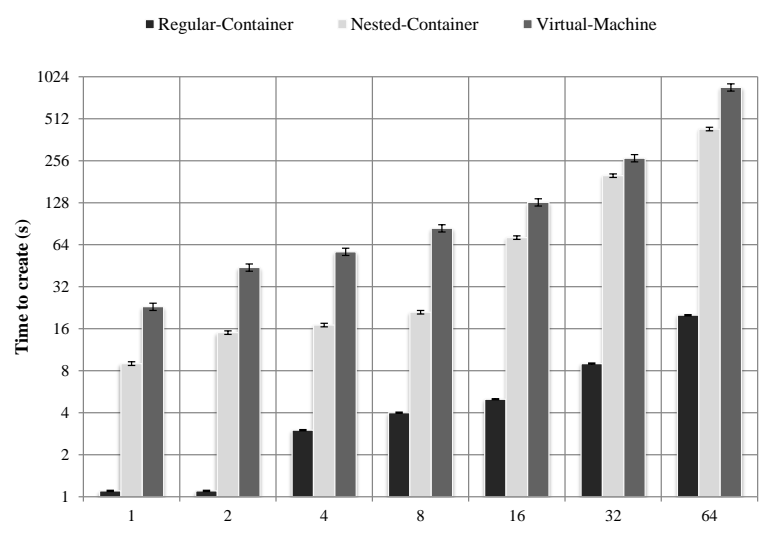

Number of instances

Fig. 4. Time to create an increasing number of instances of virtual containers (base $2 \log$ scale in both axes). Where the nested-container is a fully initialized parent plus one child.

The results of our experiments are available in Figure 4, which shows the measured time to create different number of instances under each environment. As expected regular containers are always the fastest approach, followed by nestedcontainers and virtual machines. While the creation of a single nested-container has almost 8 times more overhead than the creation of one regular container, the creation of nestedcontainers is still more than twice as fast as virtual machines.

This additional overhead for nested-containers is related to the initialization of Docker in the parent container, which also involves loading an image stored locally on the host and the creation of the child container itself. The main overhead is related to the image loading, which takes in average 6.2s. In order to avoid this loading time, a parent container can be created with a shared pre-created (read-only) volume already contain the child image loaded. We verified this approach creating a parent plus a child container with the parent sharing a preloaded volume, and the creation time dropped from 8 s to $1.7 \mathrm{~s}$. However, such approach has some drawbacks since many parents will be sharing the same volume, such as concurrency and security problems (especially the last one, since the parent is a privileged container). Because of this trade-off, our focus in this paper is to show the overhead to fully initialize a parent container.

Also, note that when creating more than 8 nestedcontainers, the overall creation time seems to increase more than linearly, and becomes a lot closer to virtual machines than regular containers. The host machine only has 12 cores, so the behavior when overloading the cores is significantly different. However, nested-containers are still twice as fast as virtual machines in most scenarios.

\section{Experiment 3: Overhead of Nested-Container Creation}

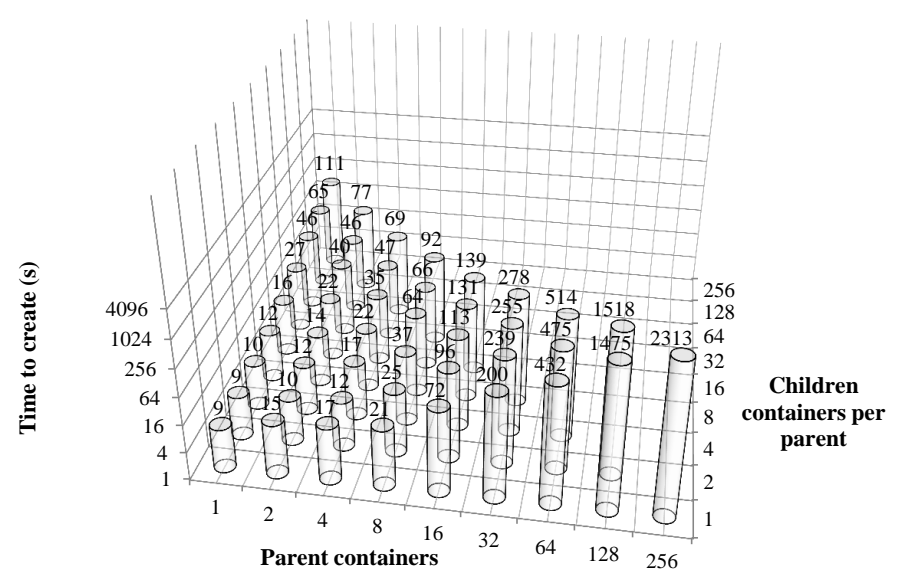

Fig. 5. Time to create nested-containers with different ratios of fully initialized parent and child containers (base 2 log scale)

Following Experiment 2, which evaluates the creation of parent containers with a single child container, this Section focuses on the overhead of creating parents with multiple children.

The goal of this experiment is to measure the overhead of different ratios of parent to child containers. In each execution, we change the number of concurrent parent containers, from 1 up to 256 , also varying the number of concurrent children in each parent, again from 1 up to 256. The maximum number of concurrent children running at a time is 256 . We measure the elapsed time, from start-up to exiting the parent container, which involves starting the Docker daemon, loading a locallystored image, starting-up all children containers, and exiting all of them.

The results of this experiment are summarized in Figure 5 which shows the time to create nested-containers with different configurations of parent to child containers (for clarity, we also include the same average times as well as the standard deviation in Table I). As it can be observed, the creation of only one parent with 256 children takes 111 seconds to complete. However, 256 parents with only one child takes 2312 seconds. This is due to the bottleneck's relation to the initialization of the parent container, which is supposed to initialize Docker and load children image. Once the parent is running, the creation of child containers does not take more than the creation of a regular container, and will be faster than the creation of new parents. Moreover, the results show that with less than 
TABLE I. TIME TO CREATE NESTED-CONTAINERS WITH DIFFERENT RATIOS OF PARENT TO CHILDREN CONTAINERS

\begin{tabular}{|c|c|c|c|c|c|c|c|c|c|}
\hline \# Child \# Parent & 1 & 2 & 4 & 8 & 16 & 32 & 64 & 128 & 256 \\
\hline 1 & $9 \mathrm{~s} \sigma=1.0$ & $15 \mathrm{~s} \sigma=0.5$ & $17 \mathrm{~s} \sigma=0.5$ & $21 \mathrm{~s} \sigma=0.4$ & $72 \mathrm{~s} \sigma=1.3$ & $200 \mathrm{~s} \sigma=0.3$ & $432 \mathrm{~s} \sigma=10.5$ & $1475 \mathrm{~s} \sigma=146.5$ & 2313 s $\sigma=160.8$ \\
\hline 2 & $9 \mathrm{~s} \sigma=0.5$ & $10 \mathrm{~s} \sigma=0.51$ & $12 \mathrm{~s} \sigma=0.43$ & $25 \mathrm{~s} \sigma=2.0$ & $96 \mathrm{~s} \sigma=4.0$ & $239 \mathrm{~s} \sigma=2.5$ & $475 \mathrm{~s} \sigma=14.5$ & $1518 \mathrm{~s} \sigma=163.0$ & - \\
\hline 4 & $10 \mathrm{~s} \sigma=0.5$ & $12 \mathrm{~s} \sigma=0.5$ & $17 \mathrm{~s} \sigma=0.5$ & $37 \mathrm{~s} \sigma=2.0$ & $113 \mathrm{~s} \sigma=8.0$ & 255 s $\sigma=0.6$ & $514 \mathrm{~s} \sigma=12.0$ & - & - \\
\hline 8 & $12 \mathrm{~s} \sigma=0.5$ & $14 \mathrm{~s} \sigma=0.53$ & $22 \mathrm{~s} \sigma=3.56$ & $64 \mathrm{~s} \sigma=1.5$ & $131 \mathrm{~s} \sigma=6.1$ & $278 \mathrm{~s} \sigma=7.0$ & - & - & - \\
\hline 16 & $16 \mathrm{~s} \sigma=1.0$ & $22 \mathrm{~s} \sigma=1.0$ & $35 \mathrm{~s} \sigma=1.5$ & $66 \mathrm{~s} \sigma=0.5$ & $139 \mathrm{~s} \sigma=1.5$ & - & - & - & - \\
\hline 32 & $27 \mathrm{~s} \sigma=1.5$ & $40 \mathrm{~s} \sigma=0.5$ & 47 s $\sigma=4.81$ & $92 \mathrm{~s} \sigma=3.5$ & - & - & - & - & - \\
\hline 64 & $46 \mathrm{~s} \sigma=2.0$ & 46 s $\sigma=2.0$ & $69 \mathrm{~s} \sigma=2.02$ & - & - & - & - & - & - \\
\hline 128 & $65 \mathrm{~s} \sigma=3.0$ & $77 \mathrm{~s} \sigma=1.0$ & - & - & - & - & - & - & - \\
\hline 256 & $111 \mathrm{~s} \sigma=3.5$ & - & - & - & - & - & - & - & - \\
\hline
\end{tabular}

16 parents, the creation of 256 child containers benefits from the concurrent child's creation across different parents. The creation of 256 children into a single parent takes 111 seconds, while the creation of the same number of children across 4 parents only takes 69 seconds. When we are overloading the host machine with more parents than available cores, the creation time is even higher (more than 139 seconds).

\section{E. Experiment 4: Network Performance - Local traffic in one host}

As described in Section II, microservices architectures may involve multiple applications communicating with the same machine. The goal of this experiment is to measure network performance, with a focus on studying the communication overhead of different technologies when running on the same host.

In order to evaluate the network performance, we select the Netperf benchmark [24]. In particular, the Netperf tests used to evaluate performance are TCP Stream (TCP_STREAM) and TCP Request/Response (TCP_RR). TCP_STREAM is a simple test that transfers a certain amount of data from a client running netperf to a server running netserver. This test calculates the throughput and does not include the time to establish the connection. On the other hand, TCP_RR is a synchronous test that consists of exchanging requests and responses (transactions), and which can be used to infer oneway and round-trip latencies. In particular, $T C P \_R R$ executions were configured to run in burst mode in order to have more than one transaction at the same time, and the socket buffer size of connection data set to $256 \mathrm{~K}$. Throughput and round trip latency per transaction were measured with Netperf under different environments: bare-metal, containers, and virtual machines; and also under different network virtualization technologies, such as Host-Network, OpenvSwitch, and Linux Bridge.

In this experiment, both server and client (netserver and netperf respectively) were executed in different configurations: host to host, container to host, host to container, virtual machine to host, host to virtual machine. Additionally, each environment can be configured with different network virtualization technologies, such as Linux Bridge or OpenvSwitch [25]. Containers might also be configured as HostNetwork where there is no virtualization layer and containers use the native host network stack directly. While Host-Network might provide certain performance improvement, it also has security implications since it doesn't provide any network isolation. In this experiment, OpenvSwitch is configured only for routing packets and there is no additional encapsulation, while
Linux Bridge is combined with forwarding NAT iptable rules. Since Docker is not yet fully integrated with OpenvSwitch, it requires additional configuration, which involves creating a virtual interface (veth pairs), binding one in the OpenvSwitch bridge and another one in an already started container.

The results of this experiment are presented in Table II. All executions were repeated 5 times, and the table includes average time and standard deviation. In this experiment, it should also be noted that, even though the host is composed of a single NIC of 1 Gigabit, the throughput is higher than $1 \mathrm{Gbps}$. Since the client and the server are running on the same host, the network packets are not going through the NIC, they are going to the loopback interface. The bottleneck is the CPU throughput.

As it can be observed in Table II, the network performance when using containers is generally higher than that of virtual machines, and it can be as fast as bare-metal under certain configurations. For instance, when the containers are configured with Host-Network, they basically display the same performance as bare-metal in terms of both, throughput and latency. On the other hand, when containers or virtual machines are configured with Linux Bridge or OpenvSwitch, there is a significant performance impact. Even though OpenvSwitch is supposed to achieve higher performance than Linux Bridge, as stated in [26], our results show that their behavior in terms of throughput and latency under these configurations is similar, and their performance is not significantly different, only achieving approximately half of the throughput, and almost twice as much latency. Finally, it should also be noted that even if virtual machines are accelerated to provide high computing performance, the network still lacks behind in terms of performance when compared to containers, and it's approximately twice as slow, even when compared against the same network virtualization technologies.

TABLE III. NETWORK THROUGHPUT AND LATENCY EVALUATION OF NESTED-CONTAINERS

\begin{tabular}{|c|c|c|c|}
\hline $\begin{array}{c}\text { Parent } \\
\text { (privileged) }\end{array}$ & Child & Throughput & Latency \\
\hline \hline Host-network & Host-network & 33.74 Gbps $\sigma=2.3$ & $109.31 \mu s \quad \sigma=7.66$ \\
\hline Host-network & Linux Bridge & 16.06 Gbps $\sigma=0.73$ & $228.90 \mu s \quad \sigma=10.52$ \\
\hline Linux Bridge & Host-network & $15.56 \mathrm{Gbps} \sigma=0.97$ & $236.72 \mu s \quad \sigma=14.72$ \\
\hline Linux Bridge & Linux Bridge & $12.53 \mathrm{Gbps} \sigma=0.75$ & $293.84 \mu s \quad \sigma=18.45$ \\
\hline Open vSwitch & Host-network & $16.9 \mathrm{Gbps} \sigma=0.44$ & $217.25 \mu s \quad \sigma=5.63$ \\
\hline Open vSwitch & Linux Bridge & $12.19 \mathrm{Gbps} \sigma=0.57$ & $301.54 \mu s \quad \sigma=14.63$ \\
\hline
\end{tabular}

In addition to studying the performance of different virtualization environments, in Table III we also show the result of evaluating network throughput and latency of nested-containers under different combinations of network configurations for par- 
TABLE II. NETWORK THROUGHPUT AND LATENCY EVALUATION FOR DIFFERENT CONFIGURATIONS OF CLIENT/SERVER UNDER BARE-METAL, CONTAINER AND VIRTUAL MACHINE ON A SINGLE HOST MACHINE

\begin{tabular}{|c|c|c|c|c|c|c|}
\hline & \multicolumn{3}{|c|}{ Throughput } & \multicolumn{3}{|c|}{ Latency } \\
\hline (Client - Server) & Host-Network & Linux Bridge & Open vSwitch & Host-Network & Linux Bridge & Open vSwitch \\
\hline Host - Host & 35.71 Gbps $\sigma=0.32$ & - & - & $102.77 \mu s \quad \sigma=0.95$ & - & - \\
\hline Container - Host & 35.13 Gbps $\sigma=0.48$ & 15.82 Gbps $\sigma=0.36$ & 16.01 Gbps $\sigma=0.47$ & $104.48 \mu s \quad \sigma=1.45$ & $231.97 \mu s \quad \sigma=5.3$ & $229.37 \mu s \quad \sigma=6.38$ \\
\hline Host - Container & 34.96 Gbps $\sigma=0.63$ & 15.96 Gbps $\sigma=0.51$ & 16.86 Gbps $\sigma=0.35$ & $105.0 \mu s \quad \sigma=1.94$ & $230.17 \mu s \sigma=7.35$ & $217.76 \mu s \quad \sigma=4.63$ \\
\hline Virtual machine - Host & - & 8.64 Gbps $\sigma=0.28$ & 7.94 Gbps $\sigma=0.69$ & - & $424.92 \mu s \quad \sigma=14.09$ & $465.53 \mu s \quad \sigma=43.57$ \\
\hline Host - Virtual machine & - & 9.24 Gbps $\sigma=0.27$ & 8.77 Gbps $\sigma=0.55$ & - & $397.53 \mu s \quad \sigma=12.08$ & $420.14 \mu s \quad \sigma=27.09$ \\
\hline
\end{tabular}

ent and child containers. As expected, when a parent or a child container is configured with Host-Network, the performance is significantly better than that of Linux Bridge or OpenvSwitch, which are once again approximately twice as slow in terms of both, throughput and latency. However, while Host-Network provides higher performance, it also has certain security tradeoffs, especially when using it in the parent container. Parent containers are privileged, so they might be granted access to all the packets in the host machine. Hence Host-Network is only a viable configuration when used in the child containers, since they are not allowed to access the host network. Note that in this experiment OpenvSwitch couldn't be used in the child container due to privilege-related issues, so OpenvSwitch parent containers are only compared against Host-Network and Linux bridge child containers.

\section{F. Experiment 5: Network Performance - Remote traffic across two hosts}

This experiment is similar to the previous experiment described in section IV-E, except network performance is measured across two hosts interconnected by a physical 1 Gigabit switch.

Netperf tests are once again TCP_STREAM and TCP_RR with the same configuration. However, in this experiment, netserver and the netperf client runs on different machines. The server runs directly on bare-metal in one machine and the client runs in all the studied environments: baremetal, containers, and virtual machines. As in the previous experiment we also evaluate different network virtualization technologies, including Linux Bridge, OpenvSwitch, and HostNetwork (only available for containers).

The results of this experiment are presented in Tables IV and $\mathrm{V}$. The former is focused on comparing network performance of bare-metal, containers, and virtual machines, while the latter compares different configurations of nestedcontainers. All executions were repeated 5 times, and the tables include average time and standard deviation for each configuration.

As it can be observed in Table IV, throughput is significantly lower and latency is higher in this experiment (from Gbps and $\mu s$ in the previous experiment to Mbps and $m s$ here). However, one of the consequences of using the physical network is that the bottleneck is now the physical network itself, and the performance of different configurations of virtual containers and network virtualization technologies is not as varied. The major difference is latency when using virtual machines, which is approximately $12 \%$ higher. This is due to virtual machine simulates the entire network stack in the guest operating system, which involves additional overhead when compared with containers.
TABLE V. NETWORK THROUGHPUT AND LATENCY EVALUATION OF NESTED-CONTAINERS CONNECTED TO A REMOTE HOST

\begin{tabular}{|c|c|c|c|}
\hline $\begin{array}{c}\text { Parent } \\
\text { (privileged) }\end{array}$ & Child & Throughput & Latency \\
\hline \hline Host-network & Host-network & $148.98 \mathrm{Mbps} \sigma=5.33$ & $24.72 \mathrm{~ms} \sigma=0.92$ \\
\hline Host-network & Linux Bridge & $147.39 \mathrm{Mbps} \sigma=4.78$ & $24.98 \mathrm{~ms} \sigma=0.83$ \\
\hline Linux Bridge & Host-network & $147.29 \mathrm{Mbps} \sigma=4.85$ & $25.01 \mathrm{~ms} \sigma=0.81$ \\
\hline Linux Bridge & Linux Bridge & $148.31 \mathrm{Mbps} \sigma=4.79$ & $24.83 \mathrm{~ms} \sigma=0.81$ \\
\hline Open vSwitch & Host-network & $147.75 \mathrm{Mbps} \sigma=5.75$ & $24.93 \mathrm{~ms} \sigma=0.97$ \\
\hline Open vSwitch & Linux Bridge & $147.86 \mathrm{Mbps} \sigma=4.99$ & $24.91 \mathrm{~ms} \sigma=0.85$ \\
\hline
\end{tabular}

Table V summarizes the evaluation of nested-containers under different combinations of network configurations for parent and child containers. Again, once the physical network becomes the bottleneck there is no significant performance impact on comparing different combinations and configurations of network virtualization technologies. But as described in Section IV-E, Host-Network still poses security concerns when executed on the parent container.

\section{CONCLUSIONS}

Containers are gaining momentum because they offer lightweight OS virtualization capabilities. They are commonly used to host single processes in isolation on the system. While they offer clear advantages in terms of lightness and performance under several circumstances, they show limitations from the infrastructure management perspective. On the other hand, Server Virtualization has been widely adopted across sectors and industries because it provides simple mechanisms to manage the infrastructure, and group processes and applications. But it introduces several significant penalties in terms of deployment time, memory consumption and processing overheads that vary with the nature of the applications that they host.

This paper explores the use of Related Processes Per Container (RPPC) as an abstraction to leverage Containers technology, but overcoming their limitations from the point of view of infrastructure management and application deployment. RPPCs allow for the creation of ensembles that encapsulate sets of related processes that are related and provide sets functionalities required by an application once it is deployed. The RPPC approach typically speeds up deployment, reduces disruption and generally empowers the devops team. For this reason, RPPCs are particularly suitable for the implementation of microservices.

Through the experiments of the paper, we evaluate the performance impact of choosing between the two models for implementing RPCCs that we have presented: in the first approach ("master-slave") all child containers are peers of each other and a parent container which serves to manage the; in the second approach ("nested-container"), involves the parent 
TABLE IV. NETWORK THROUGHPUT AND LATENCY EVALUATION FOR DIFFERENT CONFIGURATIONS OF CLIENT/SERVER UNDER BARE-METAL, CONTAINER AND VIRTUAL MACHINE ACROSS TWO HOSTS

\begin{tabular}{|l|c|c|c||c|c||c|}
\hline & \multicolumn{3}{|c||}{ Throughput } & \multicolumn{2}{c|}{ Latency } \\
\hline (Client - Server) & Host-Network & Linux Bridge & Open vSwitch & host-network & Linux Bridge & Open vSwitch \\
\hline \hline Host - Host & $142.21 \mathrm{Mbps} \sigma=8.64$ & - & - & $25.97 \mathrm{~ms} \sigma=1.7$ & - \\
\hline Container - Host & $157.92 \mathrm{Mbps} \sigma=1.06$ & $154.51 \mathrm{Mbps} \sigma=5.22$ & $157.25 \mathrm{Mbps} \sigma=3.95$ & $23.29 \mathrm{~ms} \sigma=0.15$ & $23.83 \mathrm{~ms} \sigma=0.82$ & $23.40 \mathrm{~ms} \sigma=0.58$ \\
\hline Virtual machine - Host & - & $135.92 \mathrm{Mbps} \sigma=6.77$ & $136.92 \mathrm{Mbps} \sigma=5.37$ & - & $27.13 \mathrm{~ms} \sigma=1.31$ & $26.5 \mathrm{~ms} \sigma=1.23$ \\
\hline
\end{tabular}

container being a privileged container and the child containers being in its namespace.

Our results show that the nested-containers approach is a suitable model, thanks to improved resource sharing (same memory and disk), easily performing IPC and guaranteeing fate sharing among the containers in the same nested-container. The results show that nested-containers don't have a significant impact on the performance of CPU, however, there are some trade-offs in terms of network performance compared to baremetal and regular containers. In any case, they add some of the simplicity that Virtual Machines offer in terms of infrastructure management flexibility and ease of workload deployment.

Finally, it might be worth to consider the trade-off when selecting the most appropriated model to implement microservices architecture relying on network performance, security and simplicity. In our future work, we are planning to implement an extension on Docker to fully support OVS and study the performance of a real application implementation based on microservices architecture using nested-containers. Additionally, we will study the overhead related to the control plane.

\section{ACKNOWLEDGMENTS}

This project is supported by the IBM/BSC Technology Center for Supercomputing collaboration agreement. It has also received funding from the European Research Council (ERC) under the European Union's Horizon 2020 research and innovation programme (grant agreement No 639595). It is also partially supported by the Ministry of Economy of Spain under contracts TIN2012-34557 and 2014SGR1051 and by the BSC-CNS Severo Ochoa program (SEV2011-00067).

\section{REFERENCES}

[1] M. Kerrisk, "Control group configuration unit settings," accessed in: 29-January-2015. [Online]. Available: http://man7.org/linux/manpages/man5/systemd.cgroup.5.html

[2] - "Control group configuration unit settings," accessed in: 29-January-2015. [Online]. Available: http://man7.org/linux/manpages/man7/systemd.namespaces.7.html

[3] OpenVZ, accessed in: 21-January-2015. [Online]. Available: http://openvz.org/main_page

[4] Docker, "What is docker?" accessed in: 21-January-2015. [Online] Available: https://www.docker.com/

[5] W. Felter, a. Ferreira, R. Rajamony, and R. J., "An updated performance comparison of virtual machines and linux containers," IBM Research Division, Tech. Rep. RC25482, 2014.

[6] Rocket, "Rocket - app container runtime," accessed in: 25-March-2015. [Online]. Available: https://github.com/coreos/rocket

[7] T. Erl, Service-Oriented Architecture: Concepts, Technology, and Design. Upper Saddle River, NJ, USA: Prentice Hall PTR, 2005.

[8] D. Namiot and M. Sneps-Sneppe, "On micro-services architecture," International Journal of Open Information Technologies, vol. 2, no. 9, 2014.
[9] J. Lewis and M. Fowler, "Microservices," 2014, accessed in: 21-January-2015. [Online]. Available: $\mathrm{http} / / /$ martinfowler.com/articles/microservices.html

[10] N. Huber, M. v. Quast, M. Hauck, and S. Kounev, "Evaluating and modeling virtualization performance overhead for cloud environments," CLOSER, pp. 563-573, 2011.

[11] J. Hwang, S. Zeng, F. Wu, and T. Wood, "A component-based performance comparison of four hypervisors." Integrated Network Management (IM 2013), 2013 IFIP/IEEE International Symposium, pp. 269-276.

[12] R. J. Creasy, The origin of the VM/370 time-sharing system. IBM Journal of Research and Development,, 1981, no. (5), ch. 25, pp. 483490.

[13] S. Soltesz, H. Potzl, M. E. Fiuczynski, A. Bavier, and L. Peterson, "Container-based operating system virtualization: A scalable, highperformance alternative to hypervisors," in Proceedings of the 2nd ACM SIGOPS/EuroSys European Conference on Computer Systems, 2007, pp. 275-287.

[14] IBM, "Ibm," accessed in: 24-March-2015. [Online]. Available: https://developer.ibm.com/bluemix/2014/12/04/ibm-containersbeta-docker/

[15] — , "Ibm," accessed in: 24-March-2015. [Online]. Available: https://console.ng.bluemix.net/solutions/open-architecture-containers

[16] R. Dua, A. R. Raja, and D. Kakadia, "Virtualization vs containerization to support paas," in Proceedings of the 2014 IEEE International Conference on Cloud Engineering, ser. IC2E '14. Washington, DC, USA: IEEE Computer Society, 2014, pp. 610-614. [Online]. Available: http://dx.doi.org/10.1109/IC2E.2014.41

[17] M. Xavier, M. Neves, F. Rossi, T. Ferreto, T. Lange, and C. De Rose, "Performance evaluation of container-based virtualization for high performance computing environments," in Parallel, Distributed and Network-Based Processing (PDP), 2013 21st Euromicro International Conference on, Feb 2013, pp. 233-240.

[18] M. Casoni, C. Grazia, and N. Patriciello, "On the performance of linux container with netmap/vale for networks virtualization," in Networks (ICON), 2013 19th IEEE International Conference on, Dec 2013, pp. $1-6$.

[19] Google, "Google," accessed in: 24-March-2015. [Online]. Available: https://cloud.google.com/container-engine/docs/pods/operations

[20] — "Kubernetes," accessed in: 21-January-2015. [Online]. Available: https://github.com/googlecloudplatform/kubernetes

[21] Mesos, "Mesos," accessed in: 25-March-2015. [Online]. Available: http://mesos.apache.org

[22] B. Hinfman, A. Konwinski, M. Zaharia, A. Ghodsi, A. D. Joseph, S. Shenker, and I. Stoica, "Mesos: A platform for fine-grained resource sharing in the data center," University of California Berkeley, Tech. Rep., 2010.

[23] A. Kopytov, "Sysbench manual," 2009, accessed in: 21-January-2015. [Online]. Available: http://imysql.com/wpcontent/uploads/2014/10/sysbench-manual.pdf

[24] R. Jones. Hewlett-Packard Company, 2012, accessed in: 24-March-2015. [Online]. Available: http://www.netperf.org/svn/netperf2/trunk/doc/netperf.pdf

[25] OpenvSwitch, accessed in: 21-January-2015. [Online]. Available: http://openvswitch.org/

[26] F. Callegati, W. Cerroni, C. Contoli, and G. Santandrea, "Performance of network virtualization in cloud computing infrastructures: The openstack case," in Cloud Networking (CloudNet), 2014 IEEE 3rd International Conference on, Oct 2014, pp. 132-137. 